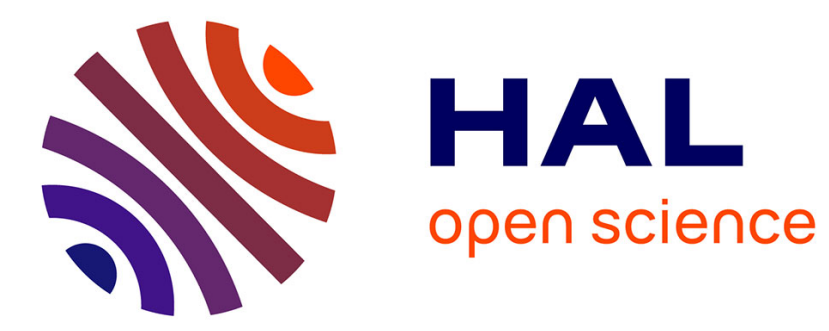

\title{
The Billard Theorem for Multiple Random Fourier Series
}

\author{
Samuel Ronsin, Hermine Biermé, Lionel Moisan
}

\section{To cite this version:}

Samuel Ronsin, Hermine Biermé, Lionel Moisan. The Billard Theorem for Multiple Random Fourier Series. Journal of Fourier Analysis and Applications, 2017, 23 (1), pp.207-228. 10.1007/s00041-0169467-9 . hal-00850112v2

\section{HAL Id: hal-00850112 \\ https://hal.science/hal-00850112v2}

Submitted on 24 Oct 2016

HAL is a multi-disciplinary open access archive for the deposit and dissemination of scientific research documents, whether they are published or not. The documents may come from teaching and research institutions in France or abroad, or from public or private research centers.
L'archive ouverte pluridisciplinaire HAL, est destinée au dépôt et à la diffusion de documents scientifiques de niveau recherche, publiés ou non, émanant des établissements d'enseignement et de recherche français ou étrangers, des laboratoires publics ou privés. 


\title{
THE BILLARD THEOREM FOR MULTIPLE RANDOM FOURIER SERIES
}

\author{
SAMUEL RONSIN $^{1}$, HERMINE BIERMÉ ${ }^{2}$, AND LIONEL MOISAN ${ }^{1}$
}

\begin{abstract}
We propose a generalization of a classical result on random Fourier series, namely the Billard Theorem, for random Fourier series over the $d$-dimensional torus. We provide an investigation of the independence with respect to a choice of a sequence of partial sums (or method of summation). We also study some probabilistic properties of the resulting sum field such as stationarity and characteristics of the marginal distribution.
\end{abstract}

AMS 2010 Mathematics Subject Classification. Primary: 42B05, 60G60, 60G17; Secondary: 42B08, 60 G50.

keywords: Billard Theorem; Random Fourier series; Multiple Fourier series; Random phase; Random fields.

\section{INTRODUCTION}

Random Fourier series have a long and rich history. First introduced by Paley and Zygmund in their series of papers [17] [18] and [19] in the 1930's, the subject has been drawing attention ever since. The most prominent work on the matter, along with many applications to harmonic analysis, has been synthesized by Kahane in [11] and Marcus and Pisier in [16], and many problems are still open as of today.

The purpose of this paper is to prove the equivalence between different important properties for multiple random Fourier series. In dimension 1, the celebrated Billard Theorem (as stated in Kahane's famous book [11], Theorem 3 p. 58 - the original article by Billard [3] attempts to prove a slightly weaker result) claims a chain of equivalences between almost sure continuity, uniform convergence, uniform boundedness, and pointwise convergence of random Fourier series. This chain is very surprising since it connects properties that are obviously non-equivalent under general nonprobabilistic hypotheses. One interesting point of such a result is that it allows one to define $2 \pi$ periodical processes through the law of their Fourier coefficients. Moreover, continuity is proven to hold over the pointwise limit of the series, and not over some modified processes as it is often the case in probability theory.

Interestingly, the Billard Theorem has been partially extended in different directions. The equivalence between almost sure uniform convergence and almost sure uniform boundedness for Gaussian random Fourier series is well known (see e.g. [13] Theorem 13.4). Most notably, the same equivalence for Fourier series on any compact group has been proven in [8], without assuming the Fourier coefficients to be Gaussian. However, a proof of an extension of the whole chain of equivalences to the case of Fourier series on the $d$-dimensional torus (for $d>1$ ) was missing. This paper proposes to extend the techniques introduced by Kahane in order to provide such a proof.

In order to state such an extension, we shall write our hypotheses in Section 2. In Section 3, we introduce a notion of convergence for non absolutely summable sums taken over $\mathbb{Z}^{d}$ and claim a result of independence with respect to the variations of this notion. This independence is largely based on the Itô-Nisio Theorem [10]. In Section 4, we state and prove an extension of Billard Theorem to the $d$-dimensional torus. Moreover, we discuss direct generalizations which include the Gaussian case. In Section 5, we study the law of the resulting process under the hypothesis of uniform convergence of the partial sums of the random Fourier series. 


\section{Notations And Hypotheses}

2.1. Notations. Throughout the article, we consider $(\Omega, \mathcal{F}, \mathbb{P})$ a complete probability space, and $\mathbb{T}^{d}:=\mathbb{R}^{d} / 2 \pi \mathbb{Z}^{d}$ the $d$-dimensional torus over which we consider the usual Lebesgue measure. We are interested in real stationary centered second-order processes defined on $\mathbb{T}^{d}$. Our purpose in this article is to define such processes through the law of their random Fourier representation. For any function $f$ in $L^{2}\left(\mathbb{T}^{d}\right)$ with real or complex values, let us write the Fourier coefficients

$$
\widehat{f}_{n}:=<f, e_{n}>=\int_{\mathbb{T}^{d}} f(t) e^{-i n \cdot t} d t
$$

where $e_{n}: t \mapsto e^{i n \cdot t}$ for all $n$ in $\mathbb{Z}^{d}\left(a \cdot b\right.$ denotes the canonical inner product in $\mathbb{R}^{d}$ and $<g, h>$ the canonical Hermitian inner product in $L^{2}\left(\mathbb{T}^{d}\right)$ ).

Let $X: \Omega \times \mathbb{T}^{d} \rightarrow \mathbb{R}$ denote a second-order process, i.e. such that $X(\cdot, t)$ (often written $X(t)$ in the following) is in $L^{2}(\Omega)$ for all $t$ in $\mathbb{T}^{d}$. Moreover, $X$ is assumed to be centered, i.e. such that $\mathbb{E}[X(t)]=0$ for all $t$, and weakly stationary, i.e. such that $\operatorname{Cov}(X(s), X(t))$ only depends on $t-s$. In particular, $\mathbb{E}\left[X(t)^{2}\right]<\infty$ and this quantity does not depend on $t$. Thus, thanks to Fubini-Tonelli Theorem, it follows that the sample paths of $X$ belong almost surely to the space $L^{2}\left(\mathbb{T}^{d}\right)$ of square integrable functions. Hence, for some real non-negative random variables $\left(A_{n}\right)_{n \in \mathbb{Z}^{d}}$ that are almost surely in $l^{2}\left(\mathbb{Z}^{d}\right)$ and $\left(\Phi_{n}\right)_{n \in \mathbb{Z}^{d}}$ random variables in $\mathbb{R} / 2 \pi \mathbb{Z}$, one can write

$$
X(\omega, \cdot) \stackrel{L^{2}\left(\mathbb{T}^{d}\right)}{=} \sum_{n \in \mathbb{Z}^{d}} A_{n}(\omega) e^{i \Phi_{n}(\omega)} e_{n}
$$

almost surely (recall that $\left(e_{n}\right)_{n \in \mathbb{Z}^{d}}$ is a Hilbert basis of $L^{2}\left(\mathbb{T}^{d}\right)$ ). In other words $\widehat{X}_{n}(\omega)=A_{n}(\omega) e^{i \Phi_{n}(\omega)}$.

However, (2) does not hold a priori in the sense "almost surely for all $t$ in $\mathbb{T}^{d}$ ". Thus, defining a second-order process over $\mathbb{T}^{d}$ through the law of its random Fourier coefficients is generally not straightforward. Indeed, two second-order processes $Y$ and $Z$ that have the same random Fourier representations $\left(\widehat{Y}_{n}=\widehat{Z}_{n}\right.$ for all $n$ in $\mathbb{Z}^{d}$ almost surely) do not necessarily satisfy finite-dimensional distribution equality (e.g., one could have $Y(0)=0$ a.s. and $Z(0)=1$ a.s.). Moreover, for any set of null Lebesgue-measure $\mathcal{N} \subset \mathbb{T}$, there exists a function $f$ in $\mathcal{C}^{0}(\mathbb{T})$ such that the Dirichlet sums $\sum_{n=-N}^{N} \widehat{f}_{n} e_{n}$ diverge on $\mathcal{N}$, as proven by Katznelson [12]. Thus, defining a process unambiguously only through its Fourier coefficients can turn out to be difficult.

2.2. Two Strategies to Define a Process through its Fourier Coefficients. There are several ways to overcome these difficulties. One strategy consists in restricting our study to processes with continuous sample paths, since continuous functions with identical Fourier coefficients (hence $L^{2}\left(\mathbb{T}^{d}\right)$ equivalent) are equal everywhere. Since the inclusion of $\mathcal{C}^{0}\left(\mathbb{T}^{d}\right)$ into $L^{2}\left(\mathbb{T}^{d}\right)$ is strict, we shall seek conditions for a random family of Fourier coefficients to represent a continuous function almost surely. Another advantage of this strategy is that processes with sample paths that are almost surely in $\mathcal{C}^{0}\left(\mathbb{T}^{d}\right)$ are Radon random variables, and thus there is equivalence between equality in law as random variables in the Banach space $\mathcal{C}^{0}\left(\mathbb{T}^{d}\right)$ and equality in finite-dimensional law (see Ledoux and Talagrand [13] p. 46).

Remark 1. In the following, when considering the law of a random function that is almost surely in $\mathcal{C}^{0}\left(\mathbb{T}^{d}\right)$, we shall consider its finite dimensional law or the law of the entire process indifferently.

Another strategy would be to consider the pointwise convergence of partial sums and focus on random Fourier coefficients that yield convergence everywhere almost surely. For that matter, a sequence of partial sums or "method of summation" needs to be specified. Indeed, it can be the case that for the same Fourier coefficients $\left(a_{n}\right)_{n \in \mathbb{Z}^{d}}$, a sequence of partial sums $\left(\sum_{n \in \mathcal{A}_{k}} a_{n} e_{n}\right)_{k \in \mathbb{N}}$ is convergent almost everywhere and another sequence $\left(\sum_{n \in \mathcal{B}_{k}} a_{n} e_{n}\right)_{k \in \mathbb{N}}$ is divergent on a set of positive measure. This has been pointed out by Fefferman in [7] and [6] for the case $d=2$.

2.3. The Billard Theorem in Dimension 1. In this article, we focus on random Fourier coefficients that have the following properties: 
- $\mathcal{H}_{1}$ : " $\left(A_{n}\right)_{n \in \mathbb{Z}^{d}}=\left(a_{n}\right)_{n \in \mathbb{Z}^{d}}$ is a deterministic, non-negative, even $\left(a_{-n}=a_{n}\right.$ for all $n$ in $\left.\mathbb{Z}^{d}\right)$ square summable family with $a_{0}=0 . "$

- $\mathcal{H}_{2}$ : " $\left(\Phi_{n}\right)_{n \in \mathbb{Z}^{d}}$ is a pure phase noise field, that is for all $n$ in $\mathbb{Z}^{d}, \Phi_{-n}=-\Phi_{n}$ (modulo $2 \pi$ ) almost surely, $\Phi_{n}$ has uniform distribution over $\mathbb{R} / 2 \pi \mathbb{Z}$ and $\left(\Phi_{n}\right)_{n \in \mathcal{A}}$ are independent for all $\mathcal{A} \subset \mathbb{Z}^{d}$ such that $\mathcal{A}$ and $-\mathcal{A}$ do not intersect."

Interestingly, under the hypotheses $\mathcal{H}_{1}$ and $\mathcal{H}_{2}$, the two strategies turn out to be equivalent, as we shall see in the Section 4. This generalizes the Billard Theorem (see Billard [3] and Kahane [11] p. 58), obtained in the case where $d=1$, stating that under hypotheses $\mathcal{H}_{1}$ and $\mathcal{H}_{2}$, the conditions

(i): $\omega$-a.s. convergence everywhere of the Dirichlet sums $\left(\sum_{n=-k}^{k} A_{n}(\omega) e^{i\left(n \cdot t+\Phi_{n}(\omega)\right)}\right)_{k \in \mathbb{N}}$

(ii): $\omega$-a.s. uniform convergence of the Dirichlet sums

(iii): $\omega$-a.s. boundedness of the Dirichlet sums

(iv): $\omega$-a.s. existence of a continuous function $X$ with Fourier coefficients $\left(A_{n}(\omega) e^{i\left(n t+\Phi_{n}(\omega)\right)}\right)_{n \in \mathbb{Z}}$ are equivalent.

2.4. Discussion on the hypotheses. The evenness hypothesis in $\mathcal{H}_{1}$ is equivalent to considering only real-valued processes. As we shall see in Section 5, assuming that $\left(A_{n}\right)_{n \in \mathbb{Z}^{d}}$ are deterministic is equivalent to considering only second-order processes with a deterministic autocorrelation function. We shall also consider relaxations of this hypothesis in Section 3.

Furthermore, the set of hypotheses $\mathcal{H}_{1}$ and $\mathcal{H}_{2}$ can also be interpreted as an asymptotical extension of an image processing model $(d=2)$ for texture synthesis. Indeed the random phase noise model (first introduced by van Wijk [21]) has recently drawn a lot of attention, see e.g. Galerne et al. [9]. In a nutshell, this model of texture synthesis defines a random field over the discrete 2-dimensional torus $T=\left(\mathbb{Z} / M_{1} \mathbb{Z}\right) \times\left(\mathbb{Z} / M_{2} \mathbb{Z}\right)$ by taking the inverse discrete Fourier transform of $\left(a_{n} e^{i \Phi_{n}}\right)_{n \in T}$ where the following hypotheses are satisfied:

- $\mathcal{H}_{1}^{*}$ : " $\left(a_{n}\right)_{n \in T}$ is non-negative and even $\left(a_{-n}=a_{n}\right.$ for all $n$ in $\left.T\right)$ ";

- $\mathcal{H}_{2}^{*}$ : " $\left(\Phi_{n}\right)_{n \in T}$ is a finite pure phase noise field: for all $n$ in $T, \Phi_{-n}=-\Phi_{n}$ (modulo $2 \pi$ ) almost surely, $\Phi_{n}$ has uniform distribution over $\mathbb{R} / 2 \pi \mathbb{Z}$ and $\left(\Phi_{n}\right)_{n \in \mathcal{A}}$ are independent if $\mathcal{A}$ and $-\mathcal{A}$ do not intersect."

This model is very well suited for modeling an important class of textures, namely micro-textures (see Galerne [9]). Aside from generalizing to any dimension, our analysis consists in taking into account not only band-limited signals but also signals with an arbitrary (maybe not compact) Fourier spectrum support. This allows a much greater degree of irregularity, an important feature for some classes of stationary signals.

\section{Methods of Summation in $\mathbb{Z}^{d}$}

As we shall see in the next section, one of the difficulty in extending Billard Theorem to the case where $d \geq 2$ is that there is no straightforward equivalent of the canonical Dirichlet sums. In other words, if $d \geq 2$, there is no increasing sequence of subsets of $\mathbb{Z}^{d}$, say $\left(\mathcal{A}_{k}\right)_{k \in \mathbb{N}}$, such that any other increasing sequence of subsets of $\mathbb{Z}^{d}$, say $\left(\mathcal{B}_{k}\right)_{k \in \mathbb{N}}$, is also a subsequence of $\left(\mathcal{A}_{k}\right)_{k \in \mathbb{N}}$. This has been a major difficulty for generalizing Carleson's theorem in all finite dimensions, as discussed by Ash and Welland in [1], Fefferman in [7] and [6] (see also [20]), and more broadly for the study of Fourier series in multiple dimensions. In the following, we shall focus on increasing sequences of finite and symmetrical subsets of $\mathbb{Z}^{d}$. This assumption combined with hypotheses $\mathcal{H}_{1}$ and $\mathcal{H}_{2}$ allows us to focus on real functions.

Definition 1. $\left(\mathcal{A}_{k}\right)_{k \in \mathbb{N}}$ is said to be a (symmetrical) method of summation over $\mathbb{Z}^{d}$ if

(1) for each $k, \mathcal{A}_{k}$ is a finite subset of $\mathbb{Z}^{d}$ (such that $-\mathcal{A}_{k}=\mathcal{A}_{k}$ );

(2) for each $k, \mathcal{A}_{k} \subset \mathcal{A}_{k+1}$;

(3) $\bigcup_{k \in \mathbb{N}} \mathcal{A}_{k}=\mathbb{Z}^{d}$.

Given a Banach space $B$, a family $\left(x_{n}\right)_{n \in \mathbb{Z}^{d}}$ of elements in $B$ is said to be summable according to $\left(\mathcal{A}_{k}\right)_{k \in \mathbb{N}}$ if $\sum_{n \in \mathcal{A}_{k}} x_{n}$ converges in $B$ as $k \rightarrow \infty$.

Remark 2. A method of summation can be seen as a subsequence of an ordering sequence over $\mathbb{Z}^{d}$. 
Let us consider $\left(\mathcal{C}^{0}\left(\mathbb{T}^{d}\right),\|\cdot\|_{\infty}\right)$ the Banach space of all continuous functions over $\mathbb{T}^{d}$ endowed with the uniform convergence topology.

Remark 3. Under Hypothesis $\mathcal{H}_{2}$, for each $n \neq 0$ in $\mathbb{Z}^{d}, t \mapsto \cos \left(n \cdot t+\Phi_{n}\right)$ is a symmetrically distributed random variable in $\mathcal{C}^{0}\left(\mathbb{T}^{d}\right)$. So Hypothesis $\mathcal{H}_{2}$ implies that for any symmetrical method of summation $\left(\mathcal{A}_{k}\right)_{k \in \mathbb{N}}$, the incremental partial sums $t \mapsto \sum_{n \in \mathcal{A}_{k+1} \backslash \mathcal{A}_{k}} a_{n} e^{i\left(n \cdot t+\Phi_{n}\right)}$ are independent and symmetrically distributed.

The following result builds upon this remark and allows us to overcome the difficulties that arise with sums over $\mathbb{Z}^{d}$.

Proposition 1. Let $\left(\mathcal{A}_{k}\right)_{k \in \mathbb{N}}$ be a symmetrical method of summation in $\mathbb{Z}^{d}$. Assume that, almost surely, the sequence of functions

$$
S_{\mathcal{A}_{k}}: t \mapsto \sum_{n \in \mathcal{A}_{k}} a_{n} e^{i\left(n \cdot t+\Phi_{n}\right)}
$$

converges uniformly (resp. is uniformly bounded) on $\mathbb{T}^{d}$ as $k \rightarrow \infty$ and call $S_{\mathcal{A}}$ its limit. Then, under the hypotheses $\mathcal{H}_{1}$ and $\mathcal{H}_{2}$, for any other method of summation $\left(\mathcal{B}_{k}\right)_{k \in \mathbb{N}}$, the sequence of functions

$$
S_{\mathcal{B}_{k}}: t \mapsto \sum_{n \in \mathcal{B}_{k}} a_{n} e^{i\left(n \cdot t+\Phi_{n}\right)}
$$

converges uniformly to $S_{\mathcal{A}}$ (resp. is uniformly bounded) on $\mathbb{T}^{d}$ as $k \rightarrow \infty$.

Proof. We first prove the claim for uniform convergence. Notice that each sum over a symmetrical subset $\mathcal{E} \subset \mathbb{Z}^{d}$ such that $0 \notin \mathcal{E}$ satisfies

$$
\sum_{n \in \mathcal{E}} a_{n} e^{i\left(n \cdot t+\Phi_{n}\right)}=\sum_{n \in \mathcal{E}} a_{n} \cos \left(n \cdot t+\Phi_{n}\right)
$$

for every $t$ in $\mathbb{T}^{d}$, thanks to $\mathcal{H}_{1}$ and $\mathcal{H}_{2}$. Moreover, thanks to $\mathcal{H}_{2}, t \mapsto \cos \left(n \cdot t+\Phi_{n}\right)$ are symmetrical random variables and thus so are $t \mapsto \sum_{n \in \mathcal{E}} a_{n} e^{i\left(n \cdot t+\Phi_{n}\right)}$. Hence, for each $k \geq 2, S_{\mathcal{A}_{k}}=S_{\mathcal{A}_{0}}+$ $\sum_{p=0}^{k-1} S_{\mathcal{A}_{p+1} \backslash \mathcal{A}_{p}}$ is a sum of independent symmetrical random variables in the Banach space $\mathcal{C}^{0}\left(\mathbb{T}^{d}\right)$.

Proposition 1 can be deduced from a well known consequence of the Lévy-Itô-Nisio Theorem (see [10] or [13]) that we recall here. If $\left(Y_{k}\right)_{k \in \mathbb{N}}$ is a sequence of independent symmetrical random variables in some Banach space $(B,\|\cdot\|)$, and if $S_{k}$ denotes $\sum_{l=1}^{k} Y_{l}$, then (see e.g. [13] p. 48 and Theorem 1 in [11] p. 13), the following conditions are equivalent:

(i) $\left(S_{k}\right)_{k \in \mathbb{N}}$ converges almost surely

(ii) $\left(S_{k}\right)_{k \in \mathbb{N}}$ converges in probability

(iii) there exists some subsequence $\left(S_{k_{p}}\right)_{p \in \mathbb{N}}$ that converges almost surely.

We apply this result to the Banach space of continuous function $\left(\mathcal{C}^{0}\left(\mathbb{T}^{d}\right),\|\cdot\|_{\infty}\right)$.

Notice that, since $\mathcal{A}_{k} \subset \mathcal{A}_{k+1}$ and $\bigcup_{k \in \mathbb{N}} \mathcal{A}_{k}=\mathbb{Z}^{d}$, for any finite subset $\mathcal{E} \subset \mathbb{Z}^{d}$ one has $\mathcal{E} \subsetneq \mathcal{A}_{k}$ for $k$ large enough. Let us define a new method of summation $\left(\mathcal{A B}_{k}\right)_{k \in \mathbb{N}}$ by induction. $\mathcal{A B}_{0}=$ $\mathcal{A}_{0}, \mathcal{A B}_{1}=\bigcap_{l, \mathcal{A B}_{0} \varsubsetneqq \mathcal{B}_{l}} \mathcal{B}_{l}, \mathcal{A B}_{2}=\bigcap_{l, \mathcal{A B}_{1} \varsubsetneqq \mathcal{A}_{l}} \mathcal{A}_{l}$, and by induction $\mathcal{A B}_{2 k}=\bigcap_{l, \mathcal{A B}_{2 k-1} \varsubsetneqq \mathcal{A}_{l}} \mathcal{A}_{l}$ (resp. $\left.\mathcal{A B}_{2 k+1}=\bigcap_{l, \mathcal{A B}_{2 k} \mp \mathcal{B}_{l}} \mathcal{B}_{l}\right)$ for all $k$ in $\mathbb{N}$. Notice that this reasoning provides us $\left(p_{k}\right)_{k \in \mathbb{N}}$ and $\left(q_{k}\right)_{k \in \mathbb{N}}$, two strictly increasing sequences of integers such that $\mathcal{A B}_{2 k}=\mathcal{A}_{p_{k}}$ and $\mathcal{A B}_{2 k+1}=\mathcal{B}_{q_{k}}$. Moreover, $\left(\mathcal{A B}_{k}\right)_{k \in \mathbb{N}}$ is clearly a symmetrical method of summation.

Since $\left(S_{\mathcal{A} \mathcal{B}_{2 k}}\right)_{k \in \mathbb{N}}$ is a subsequence of $\left(S_{\mathcal{A}_{k}}\right)_{k \in \mathbb{N}}$, it converges almost surely in $\mathcal{C}^{0}\left(\mathbb{T}^{d}\right)$ to $S_{\mathcal{A}}$. Hence, thanks to the consequence of Lévy-Itô-Nisio Theorem mentioned earlier, $\left(S_{\mathcal{A} \mathcal{B}_{k}}\right)_{k \in \mathbb{N}}$ converges also almost surely in $\mathcal{C}^{0}\left(\mathbb{T}^{d}\right)$ to $S_{\mathcal{A}}$ thanks to the uniqueness of limits. It follows that $\left(S_{\mathcal{A} \mathcal{B}_{2 k+1}}\right)_{k \in \mathbb{N}}$ converges also almost surely in $\mathcal{C}^{0}\left(\mathbb{T}^{d}\right)$ to $S_{\mathcal{A}}$, as a subsequence of $\left(S_{\mathcal{A B}}\right)_{k \in \mathbb{N}}$. Thus, since $\left(S_{\mathcal{A} \mathcal{B}_{2 k+1}}\right)_{k \in \mathbb{N}}$ is a subsequence of $\left(S_{\mathcal{B}_{k}}\right)_{k \in \mathbb{N}}$, the latter converges also almost surely to $S_{\mathcal{A}}$ in $\mathcal{C}^{0}\left(\mathbb{T}^{d}\right)$, thanks to the same consequence of Lévy-Itô-Nisio Theorem. Thus $\left(S_{\mathcal{A}_{k}}\right)_{k \in \mathbb{N}},\left(S_{\mathcal{B}_{k}}\right)_{k \in \mathbb{N}}$ and $\left(S_{\mathcal{A B}_{k}}\right)_{k \in \mathbb{N}}$ converge simultaneously to the same limit almost surely. 
The proof for boundedness uses a slightly different consequence of the Lévy-Itô-Nisio Theorem. Namely with the same hypotheses and notations, the following propositions are equivalent (see e.g. Theorem 1 in [11] p. 13):

(vi) $\left(S_{k}\right)_{k \in \mathbb{N}}$ is bounded almost surely

(v) there exists some subsequence $\left(S_{k_{p}}\right)_{p \in \mathbb{N}}$ that is bounded almost surely.

Assume that $\left(S_{\mathcal{A}_{k}}\right)_{k \in \mathbb{N}}$ is almost surely bounded in $\mathcal{C}^{0}\left(\mathbb{T}^{d}\right)$. Then, so is $\left(S_{\mathcal{A} \mathcal{B}_{2 k}}\right)_{k \in \mathbb{N}}$ as a subsequence of $\left(S_{\mathcal{A}_{k}}\right)_{k \in \mathbb{N}}$. Hence, thanks to the consequence of Lévy-Itô-Nisio mentioned earlier, $\left(S_{\mathcal{A B}_{k}}\right)_{k \in \mathbb{N}}$ is also bounded in $\mathcal{C}^{0}\left(\mathbb{T}^{d}\right)$ almost surely. Hence, $\left(S_{\mathcal{A} \mathcal{B}_{2 k+1}}\right)_{k \in \mathbb{N}}$ is also bounded in $\mathcal{C}^{0}\left(\mathbb{T}^{d}\right)$ almost surely, as a subsequence of $\left(S_{\mathcal{A} \mathcal{B}_{k}}\right)_{k \in \mathbb{N}}$. Thus, since $\left(S_{\mathcal{A} \mathcal{B}_{2 k+1}}\right)_{k \in \mathbb{N}}$ is a subsequence of $\left(S_{\mathcal{B}_{k}}\right)_{k \in \mathbb{N}}$, the latter is also bounded in $\mathcal{C}^{0}\left(\mathbb{T}^{d}\right)$ almost surely, thanks to the same consequence of Lévy-Itô-Nisio Theorem.

An important consequence of Proposition 1 is that the choice of a method of summation does not matter for the uniform convergence or for the uniform boundedness. As long as uniform convergence (resp. uniform boundedness) happens almost surely for some method of convergence, it also happens almost surely for any other method of convergence and the limit is the same.

\section{Billard'S THEOREM in ARBITRARY FINITE DIMENSION}

We can now turn to an extension of Billard's theorem to the case where $d \geq 2$ (recall that the sequence $\left(S_{\mathcal{A}_{k}}\right)_{k \in \mathbb{N}}$ has been defined by equation (3)).

Theorem 1. Under the hypotheses $\mathcal{H}_{1}$ and $\mathcal{H}_{2}$, the following conditions are equivalent:

(i) almost surely, there exists a continuous function $X$, such that $\left(a_{n} e^{i \Phi_{n}}\right)_{n \in \mathbb{Z}^{d}}$ are the Fourier coefficients of $X$;

(ii) there exists a method of summation $\left(\mathcal{A}_{k}\right)_{k \in \mathbb{N}}$ such that, almost surely, $\left(S_{\mathcal{A}_{k}}\right)_{k \in \mathbb{N}}$ converges uniformly;

(iii) for all methods of summation $\left(\mathcal{A}_{k}\right)_{k \in \mathbb{N}}$, almost surely, $\left(S_{\mathcal{A}_{k}}\right)_{k \in \mathbb{N}}$ converges uniformly;

(iv) there exists a method of summation $\left(\mathcal{A}_{k}\right)_{k \in \mathbb{N}}$ such that, almost surely, $\left(S_{\mathcal{A}_{k}}\right)_{k \in \mathbb{N}}$ is bounded;

(v) for all methods of summation $\left(\mathcal{A}_{k}\right)_{k \in \mathbb{N}}$, almost surely, $\left(S_{\mathcal{A}_{k}}\right)_{k \in \mathbb{N}}$ is bounded;

(vi) for all methods of summation $\left(\mathcal{A}_{k}\right)_{k \in \mathbb{N}}$, almost surely, for all $t$ in $\mathbb{T}^{d},\left(S_{\mathcal{A}_{k}}(t)\right)_{k \in \mathbb{N}}$ converges.

The fact that (ii) $\Leftrightarrow$ (iii) (resp. (iv) $\Leftrightarrow(v)$ ) follows from Proposition 1. Moreover, (iii) implies clearly all the other statements.

Remark 4. A somewhat weaker equivalence between boundedness and continuity, which depends on a method of summation, was proven with much more generality for any compact group instead of $\mathbb{T}^{d}$ by Figa-Talamanca in [8].

Definition 2. Under any of the equivalent conditions of Theorem 1, the limit $X$ in $\mathcal{C}^{0}\left(\mathbb{T}^{d}\right)$ is called a random phase noise (RPN) process.

The remaining of this section is dedicated to the proof of Theorem 1.

\subsection{Proof of $(v) \Rightarrow($ iii $)$.}

Proposition 2. Let $\left(Y_{k}\right)_{k \in \mathbb{N}}$ be a sequence of independent random variables with value in $\mathcal{C}^{0}\left(\mathbb{T}^{d}\right)$. Assume that

(1) for each $k \in \mathbb{N}, Y_{k}$ is symmetrically distributed i.e. $-Y_{k}$ and $Y_{k}$ have the same law;

(2) for each $k \in \mathbb{N}, Y_{k}$ is stationary i.e. $Y_{k}(\cdot-\tau)$ and $Y_{k}$ have the same law for any $\tau$ in $\mathbb{T}^{d}$;

(3) the sequence $\left(\sum_{k \leq l} Y_{k}\right)_{l \in \mathbb{N}}$ is almost surely bounded in $\mathcal{C}^{0}\left(\mathbb{T}^{d}\right)$, according to the $\|\cdot\|_{\infty}$ norm. Then, almost surely, $\left(\sum_{k \leq l} Y_{k}\right)_{l \in \mathbb{N}}$ converges in $\mathcal{C}^{0}\left(\mathbb{T}^{d}\right)$.

Proposition 2 is a straightforward extension of Proposition 13 p. 55 in [11] and the proof is postponed in Appendix. We can now prove that (v) implies (iii). Assume (v), and recall that under Hypothesis $\mathcal{H}_{2}$, Remark 3 ensures that the incremental partial sums $Y_{k}:=t \mapsto \sum_{n \in \mathcal{A}_{k+1} \backslash \mathcal{A}_{k}} a_{n} e^{i\left(n \cdot t+\Phi_{n}\right)}$ satisfy the three hypotheses of Proposition 2. Thus, for any symmetrical method of summation $\left(\mathcal{A}_{k}\right)_{k \in \mathbb{N}},\left(S_{\mathcal{A}_{k}}\right)_{k \in \mathbb{N}}$ converges almost surely in $\mathcal{C}^{0}\left(\mathbb{T}^{d}\right)$ and (iii) holds. 
The end of this section is largely built upon ideas found in Kahane [11] pp. 48 and 59-60. However, we found the details of our proof to be significantly different from the case $d=1$, so we provide them in the core of the text.

4.2. Proof of $(\boldsymbol{v i}) \Rightarrow(\boldsymbol{i v})$. To prove that (vi) implies (iv) we need to prove more intermediate results. The first one deals with trigonometric polynomials. For a trigonometric polynomial $P$ defined on $\mathbb{T}^{d}$ by

$$
P(t)=\sum_{n \in \mathcal{E}} b_{n} e^{i n \cdot t}
$$

where $b_{n} \neq 0$ is in $\mathbb{C}$ for each $n$ in the finite set $\mathcal{E} \subset \mathbb{Z}^{d}$, we define the max-degree of $P$ as

$$
d(P):=\max _{n \in \mathcal{E}}|n|_{\infty}
$$

where $|x|_{\infty}:=\max _{i}\left|x_{i}\right|$ denotes the max norm for $x$ in $\mathbb{R}^{d}$. In the following we denote $\mathbb{B}_{\infty}(t, r)=$ $\left\{s \in \mathbb{T}^{d} ;|t-s|_{\infty}<r\right\}$ the projection onto $\mathbb{T}^{d}$ of the $\mathbb{R}^{d}$ open ball of radius $r$ and center $t$ with respect to $|\cdot|_{\infty}$ onto $\mathbb{T}^{d}$.

Proposition 3. Let $\mathcal{E}$ be a finite subset of $\mathbb{Z}^{d}$ and $P(t)=\sum_{n \in \mathcal{E}} b_{n} e^{i\left(n \cdot t+\phi_{n}\right)}$ be a complex trigonometric polynomial defined on $\mathbb{T}^{d}$. Assume that there exists $q \geq 1$ in $\mathbb{N}$ and $l$ in $\mathbb{Z}^{d}$ such that $\mathcal{E} \subset l+q \mathbb{Z}^{d}$, so $t \mapsto|P(t)|$ is $\frac{2 \pi}{q}$-periodic in every direction. Assume moreover that the max-degree of $P$ is less than $K$ where $K \geq \frac{q}{2 \pi}$.

Then for every radius $\varepsilon \geq 2 \pi / q$, and center $t$ in $\mathbb{T}^{d}$, there exists $t^{\prime}$ in $\mathbb{B}_{\infty}(t, \varepsilon)$ such that

$$
\mathbb{B}_{\infty}\left(t^{\prime}, \varepsilon^{\prime}\right) \subset \mathbb{B}_{\infty}(t, \varepsilon)
$$

with $\varepsilon^{\prime} \geq(2 K)^{-1}$ and

$$
|P(s)| \geq 1 / 2\|P\|_{\infty}
$$

for all s in $\mathbb{B}_{\infty}\left(t^{\prime}, \varepsilon^{\prime}\right)$.

The proof is postponed in Appendix. We now state a result of symmetrization, useful for the remainder of the proof of Theorem 1.

Lemma 1. Let $\left(a_{n}\right)_{n \in \mathbb{Z}^{d}}$ and $\left(\Phi_{n}\right)_{n \in \mathbb{Z}^{d}}$ satisfy to Hypotheses $\mathcal{H}_{1}$ and $\mathcal{H}_{2}$. Let $\left(\mathcal{A}_{k}\right)_{k \in \mathbb{N}}$ be any method of summation and $\mathcal{B}$ be a subset of $\mathbb{Z}^{d}$. Assume that there exists a random variable $T$ such that with non-zero probability (resp. almost surely) the complex-valued sequence

$$
\left(\sum_{n \in \mathcal{A}_{k} \cap \mathcal{B}} a_{n} e^{i\left(n \cdot T+\Phi_{n}\right)}\right)_{k \in \mathbb{N}}
$$

diverges. Then there exists $\mathcal{B}^{*}$ a symmetrical subset of $\mathbb{Z}^{d}$ such that with non-zero probability (resp. almost surely) the real-valued sequence

$$
\left(\sum_{n \in \mathcal{A}_{k} \cap \mathcal{B}^{*}} a_{n} e^{i\left(n \cdot T+\Phi_{n}\right)}\right)_{k \in \mathbb{N}}
$$

diverges.

We can now prove the implication (vi) $\Rightarrow(i v)$. Let us assume that (vi) holds and that (iv) does not, and let us aim at a contradiction. Let $\left(\mathcal{A}_{k}\right)_{k \in \mathbb{N}}$ be any method of summation. The sequence of partial sums $\left(S_{\mathcal{A}_{k}}\right)_{k \in \mathbb{N}}$ is not almost surely bounded in $\mathcal{C}^{0}\left(\mathbb{T}^{d}\right)$. Hence, the event

$$
E=\left\{\omega \in \Omega ;\left(S_{\mathcal{A}_{k}}(\omega)\right)_{k \in \mathbb{N}} \text { is bounded in } \mathcal{C}^{0}\left(\mathbb{T}^{d}\right)\right\}
$$

has probability less than 1 . For each $k$, define the $\sigma$-algebra $\mathcal{F}_{k}$ generated by $\left\{e^{i \Phi_{n}}\right\}_{n \in \mathcal{A}_{k}}$ and notice that $\mathcal{F}_{k} \subset \mathcal{F}_{k+1}$. The event $E$ belongs to the asymptotic $\sigma$-algebra of $\left(\mathcal{F}_{k}\right)_{k \in \mathbb{N}}$, since $E$ is independent of any finite subset of the random variables $\left(e^{i \Phi_{n}}\right)_{n \in \mathbb{Z}^{d}}$. Thanks to the independence hypothesis in 
$\mathcal{H}_{2}$, the zero-one law applies and $\mathbb{P}(E)=0$, which in turns implies that, almost surely, $\left(S_{\mathcal{A}_{k}}\right)_{k \in \mathbb{N}}$ is unbounded in $\mathcal{C}^{0}\left(\mathbb{T}^{d}\right)$.

Symmetrization. In order to obtain a contradiction, we shall construct $\mathcal{B}$ a (non-random) subset of $\mathbb{Z}^{d}$, a method of summation $\left(\mathcal{A}_{k}\right)_{k \in \mathbb{N}}$ and a random variable $T$ such that, with non-zero probability,

$$
\text { the sequence }\left(\sum_{n \in \mathcal{B} \cap \mathcal{A}_{k}} a_{n} e^{i\left(n \cdot T+\Phi_{n}\right)}\right)_{k \in \mathbb{N}} \text { does not converge as } k \rightarrow \infty \text {. }
$$

Thanks to Lemma 1, there shall exist $\mathcal{B}^{*}$ a (non-random) symmetrical subset of $\mathbb{Z}^{d}$ such that, with non-zero probability,

$$
\text { the sequence }\left(\sum_{n \in \mathcal{B}^{*} \cap \mathcal{A}_{k}} a_{n} e^{i\left(n \cdot T+\Phi_{n}\right)}\right)_{k \in \mathbb{N}} \text { does not converge as } k \rightarrow \infty \text {. }
$$

Let us consider the random Fourier coefficients $\left(\varepsilon_{n} a_{n} e^{i \Phi_{n}}\right)_{n \in \mathbb{Z}^{d}}$ where $\varepsilon_{n}=1$ whenever $n \in \mathcal{B}^{*}$ and $\varepsilon_{n}=-1$ otherwise. Thanks to $\mathcal{H}_{2}$, this family has the same law as $\left(a_{n} e^{i \Phi_{n}}\right)_{n \in \mathbb{Z}^{d}}$. Hence,

$$
S_{\mathcal{A}_{k}}: t \mapsto \sum_{n \in \mathcal{A}_{k}} a_{n} e^{i\left(n \cdot t+\Phi_{n}\right)}
$$

has the same law in the Banach space $\mathcal{C}^{0}\left(\mathbb{T}^{d}\right)$ as

$$
S_{\mathcal{A}_{k}}^{\prime}: t \mapsto \sum_{n \in \mathcal{A}_{k}} \varepsilon_{n} a_{n} e^{i\left(n \cdot t+\Phi_{n}\right)}
$$

and since $\left(S_{\mathcal{A}_{k}}\right)_{k \in \mathbb{N}}$ is assumed to converge everywhere almost surely, $\left(S_{\mathcal{A}_{k}}^{\prime}\right)_{k \in \mathbb{N}}$ shall also converge everywhere almost surely. Hence, the sum

$$
S_{\mathcal{A}_{k}}+S_{\mathcal{A}_{k}}^{\prime}: t \mapsto 2 \sum_{n \in \mathcal{B}^{*} \cap \mathcal{A}_{k}} a_{n} e^{i\left(n \cdot t+\Phi_{n}\right)}
$$

shall in turn converge everywhere almost surely. This is contradictory with (14).

Construction. Let us now build such a set $\mathcal{B}$ and a method of summation $\left(\mathcal{A}_{k}\right)_{k \in \mathbb{N}}$. Let $\mathcal{A}_{k}=\{n \in$ $\left.\mathbb{Z}^{d} ;|n|_{\infty} \leq k\right\}$. Let us define the events

$$
E_{k}^{(1)}:=\left\{\omega \in \Omega ; \sup _{j \leq k}\left\|S_{\mathcal{A}_{j}}(\omega, \cdot)\right\|_{\infty}>2\right\}
$$

for each $k$ in $\mathbb{N}$ and notice that $E_{k}^{(1)} \subset E_{k+1}^{(1)}$ for each $k$. Since almost surely, $\left(S_{\mathcal{A}_{k}}\right)_{k \in \mathbb{N}}$ is unbounded in $\mathcal{C}^{0}\left(\mathbb{T}^{d}\right), \mathbb{P}\left(E_{k}^{(1)}\right) \rightarrow 1$ as $k \rightarrow \infty$, so there is an integer $k_{1}$ such that the probability of the event $E_{k_{1}}^{(1)}$ is larger than $1 / 2$. Furthermore, whenever $\omega$ belongs to $E_{k_{1}}^{(1)}$, thanks to Proposition 3 (with $q=1, K=k_{1}$ and $\varepsilon>\pi$ so $\left.\mathbb{B}_{\infty}(t, \varepsilon)=\mathbb{T}^{d}\right)$, there exists a random ball $U_{1}(\omega)=\mathbb{B}_{\infty}\left(T_{1}(\omega), \varepsilon_{1}\right)$ with radius $\varepsilon_{1}=\left(2 k_{1}\right)^{-1}$ such that

$$
\sup _{j \leq k_{1}}\left|S_{\mathcal{A}_{j}}(\omega, t)\right|>1
$$

for all $t$ in $\mathbb{B}_{\infty}\left(T_{1}(\omega), \varepsilon_{1}\right)$. For $\omega \in \Omega \backslash E_{k_{1}}^{(1)}$ we set $U_{1}(\omega)=\mathbb{T}^{d}$. Finally, we define $\mathcal{B}_{1}=\mathcal{A}_{k_{1}}$.

Define $q_{1}=\left\lceil 2 \pi / \varepsilon_{1}\right\rceil=\left\lceil 4 \pi k_{1}\right\rceil$. Let us consider the partition of $\mathbb{Z}^{d} \backslash \mathcal{A}_{k_{1}}$ into $q_{1}^{d}$ subsets

$$
\mathcal{C}_{1, l}=\left(l+q_{1} \mathbb{Z}^{d}\right) \backslash \mathcal{A}_{k_{1}}
$$

for each $l$ in $\left\{n \in \mathbb{N}^{d} ;|n|_{\infty}<q_{1}\right\}$. For $k \geq k_{1}$, there are $\left(2 q_{1}+1\right)^{d}$ random sequences of functions $\left(S_{k}^{(1, l)}\right)_{k \in \mathbb{N}}$ defined by

$$
S_{k}^{(1, l)}(t):=\sum_{n \in \mathcal{A}_{k} \cap \mathcal{C}_{1, l}} a_{n} e^{i\left(n \cdot t+\Phi_{n}\right)}
$$


for each $l$ in $\left\{n \in \mathbb{N}^{d} ;|n|_{\infty}<q_{1}\right\}$ and they satisfy

$$
\sum_{l \in\left\{n \in \mathbb{N}^{d} ;|n|_{\infty}<q_{1}\right\}} S_{k}^{(1, l)}=S_{\mathcal{A}_{k} \backslash \mathcal{A}_{k_{1}}}=S_{\mathcal{A}_{k}}-S_{\mathcal{A}_{k_{1}}}
$$

for $k>k_{1}$. Since $\left(S_{\mathcal{A}_{k} \backslash \mathcal{B}_{1}}\right)_{k \in \mathbb{N}}$ is almost surely unbounded in $\mathcal{C}^{0}\left(\mathbb{T}^{d}\right)$, so must be at least one of the sequences $\left(S_{k}^{(1, l)}\right)_{k \in \mathbb{N}}$. Thus for at least one of these sequences, say $\left(S_{k}^{\left(1, l_{1}\right)}\right)_{k \in \mathbb{N}}$,

$$
\mathbb{P}\left(\left(S_{k}^{\left(1, l_{1}\right)}\right)_{k \in \mathbb{N}} \text { is unbounded }\right)>0 .
$$

Thanks to the zero-one law, $\left(S_{k}^{\left(1, l_{1}\right)}\right)_{k \in \mathbb{N}}$ must be unbounded with probability 1 . For each $k>k_{1}$, define the event

$$
E_{k}^{(2)}:=\left\{\omega \in \Omega \mid \sup _{k_{1}<j \leq k}\left\|S_{j}^{\left(1, l_{1}\right)}(\omega, \cdot)\right\|_{\infty}>2\right\}
$$

and notice that $E_{k}^{(2)} \subset E_{k+1}^{(2)}$ for all $k \geq k_{1}$ in $\mathbb{N}$. Since $\mathbb{P}\left(E_{k}^{(2)}\right) \rightarrow 1$ as $k \rightarrow \infty$, there exists an integer $k_{2}$ (non-random) such that $\mathbb{P}\left(E_{k_{2}}^{(2)}\right)>1 / 2$. Thus, whenever $\omega$ belongs to $E_{k_{2}}^{(2)}$, thanks to Proposition 3 (invoked with $q=q_{1}, K=k_{2}$ and $\varepsilon=\varepsilon_{1}$ ) we know that $U_{1}(\omega)=\mathbb{B}_{\infty}\left(T_{1}(\omega), \varepsilon_{1}\right)$ contains a random ball $U_{2}(\omega)=\mathbb{B}_{\infty}\left(T_{2}(\omega), \varepsilon_{2}\right)$ with radius $\varepsilon_{2}=\left(2 k_{2}\right)^{-1}$ such that

$$
\sup _{k_{1}<j \leq k_{2}}\left|\sum_{n \in \mathcal{C}_{1, l_{1}} \cap \mathcal{A}_{j}} a_{n} e^{i\left(n \cdot t+\Phi_{n}\right)}\right|>1
$$

for each $t$ in $B\left(T_{2}(\omega), \varepsilon_{2}\right)$. For $\omega$ in $\Omega \backslash E_{k_{2}}^{(2)}$, we choose $U_{2}(\omega)=U_{1}(\omega)$. Finally, we choose $\mathcal{B}_{2}:=$ $\mathcal{C}_{1, l_{1}} \cap \mathcal{A}_{k_{2}}$.

Induction. By induction, using the same arguments (Proposition 3 invoked with $q=q_{p}, K=k_{p+1}$ and $\varepsilon=\varepsilon_{p}$ ), we construct

- two increasing sequences $\left(k_{p}\right)_{p \in \mathbb{N}}$ and $\left(q_{p}\right)_{p \in \mathbb{N}}$ with values in $\mathbb{N}$ and a real sequence $\left(\varepsilon_{p}\right)_{p \in \mathbb{N}}$ such that

$$
\forall p, q_{p}=\left\lceil 2 \pi / \varepsilon_{p}\right\rceil=\left\lceil 4 \pi k_{p}\right\rceil
$$

- a sequence $\left(l_{p}\right)_{p \in \mathbb{N}}$ with values in $\mathbb{Z}^{d}$ and a sequence $\left(\mathcal{B}_{p}\right)_{p \geq 1}$ of finite subsets of $\mathbb{Z}^{d}$ such that

$$
\mathcal{B}_{p+1} \subset\left(l_{p}+q_{p} \mathbb{Z}^{d}\right) \cap\left(\mathcal{A}_{k_{p+1}} \backslash \mathcal{A}_{k_{p}}\right)
$$

- a sequence of events $\left(E_{k_{p}}^{(p)}\right)_{p \in \mathbb{N}}$ with probability at least $1 / 2$ such that

$$
\forall \omega \in E_{k_{p}}^{(p)}, \sup _{k_{p} \leq j \leq k_{p+1}} \sup _{t \in \mathbb{T}^{d}}\left|\sum_{n \in \mathcal{B}_{p} \cap \mathcal{A}_{j}} a_{n} e^{i\left(n \cdot t+\Phi_{n}\right)}\right|>2
$$

- a sequence $\left(T_{p}\right)_{p \in \mathbb{N}}$ of random variables with values in $\mathbb{T}^{d}$

- a sequence of decreasing random open balls $\left(U_{p}\right)_{p \in \mathbb{N}}$ defined by either $U_{p}(\omega)=\mathbb{B}_{\infty}\left(T_{p}(\omega), \varepsilon_{p}\right)$ if $\omega \in E_{k_{p}}^{(p)}$, or $U_{p}(\omega)=U_{p-1}(\omega)$ otherwise. such that

$$
\forall \omega \in E_{k_{p}}^{(p)}, \forall t \in U_{p}(\omega), \sup _{k_{p} \leq j \leq k_{p+1}}\left|\sum_{n \in \mathcal{B}_{p} \cap \mathcal{A}_{j}} a_{n} e^{i\left(n \cdot t+\Phi_{n}\right)}\right|>1 .
$$

Let us denote $U^{*}(\omega)=\bigcap_{p} U_{p}(\omega)$. Since the sets $\left(\mathcal{B}_{p}\right)_{p \in \mathbb{N}}$ are disjoint, and thanks to $\mathcal{H}_{2}$, the events $\left(E_{k_{p}}^{(p)}\right)_{p \in \mathbb{N}}$ are independent. Moreover since $\mathbb{P}\left(E_{k_{p}}^{(p)}\right) \geq 1 / 2, \sum_{p} \mathbb{P}\left(E_{k_{p}}^{(p)}\right)=\infty$ and thanks to the Borel-Cantelli Lemma, almost surely, $\mathbb{P}\left(\lim \inf _{p} E_{k_{p}}^{(p)}\right)=1$. Hence, $\omega$-almost surely, there is one 
and only one (random) point $T^{*}(\omega)$ in $U^{*}(\omega)$. Define $\mathcal{B}=\bigcup_{p} \mathcal{B}_{p}$. By construction, almost surely, the complex sequence

$$
\left(\sum_{n \in \mathcal{B} \cap \mathcal{A}_{k}} a_{n} e^{i\left(n \cdot T^{*}+\Phi_{n}\right)}\right)_{k \in \mathbb{N}}
$$

is not Cauchy since $T^{*}(\omega)$ belongs to each $U_{p}(\omega)$ and $(29)$ holds for all $p$ in $\mathbb{N}$.

We conclude the proof of the implication $(v i) \Rightarrow(i v)$ by noticing that the method of summation $\left(\mathcal{A}_{k}\right)_{k \in \mathbb{N}}$, the random variable $T^{*}$ and the subset $\mathcal{B} \subset \mathbb{Z}^{d}$ satisfy the condition (13).

4.3. Proof of $(\boldsymbol{i}) \Rightarrow(\boldsymbol{i i})$. Define $|x|_{1}=\sum_{i=1}^{d}\left|x_{i}\right|\left(x\right.$ in $\left.\mathbb{R}^{d}\right)$ and let us choose $\mathcal{D}_{k}:=\left\{n \in \mathbb{Z}^{d} ;|n|_{1} \leq\right.$ $k\}(k$ in $\mathbb{N})$ as a method of summation. We consider the $(2 d-1)$-Cesàro means of the sequence of functions $\left(S_{\mathcal{D}_{k}}\right)_{k \in \mathbb{N}}$

$$
C_{(2 d-1, k)}^{\mathcal{D}}:=\frac{1}{\left(\begin{array}{c}
k+2 d-1 \\
k
\end{array}\right)} \sum_{l=0}^{k}\left(\begin{array}{c}
k-l+2 d-2 \\
k-l
\end{array}\right) S_{\mathcal{D}_{l}}
$$

for $k$ in $\mathbb{N}$ as introduced in [2]. One easily checks that the sums $S_{\mathcal{D}_{k+1} \backslash \mathcal{D}_{k}}$ are symmetrically distributed and independent. Moreover, notice that the sums $C_{(2 d-1, k)}^{\mathcal{D}}$ can be rewritten as

$$
C_{(2 d-1, k)}^{\mathcal{D}}=\sum_{l=0}^{k-1} b_{k, l} S_{\mathcal{D}_{l+1} \backslash \mathcal{D}_{l}}
$$

for each $k$, with $b_{k, l}:=\frac{\left(\begin{array}{c}k-l+2 d-1 \\ k-l\end{array}\right)}{\left(\begin{array}{c}k+2 d-1 \\ k\end{array}\right)}$ for $l \leq k$ (and $b_{k, l}:=0$ otherwise). The coefficients $\left(b_{k, l}\right)_{k, l \in \mathbb{N}}$ satisfy the properties of a matrix of summation, namely that $b_{k, l} \rightarrow 0$ as $l \rightarrow \infty$ and $b_{k, l} \rightarrow 1$ as $k \rightarrow \infty$ (see Kahane [11] p. 12). Since (i) implies that $C_{(2 d-1, k)}^{\mathcal{D}}$ converges uniformly as proven by Berens and $\mathrm{Xu}$ in [2]), Theorem 1 p. 13 in [11] yields that $\left(S_{\mathcal{D}_{k}}\right)_{k \in \mathbb{N}}$ converges uniformly almost surely, and thus (ii) holds.

This concludes the proof of Theorem 1.

4.4. Discussion and Extension. Our extension of the Billard Theorem can be generalized to weaker hypotheses. For instance, consider the Hypothesis

$\mathcal{H}_{1}^{* *}$ : " $\left(A_{n}\right)_{n \in \mathbb{Z}^{d}}$ is such that $\left(A_{n}\right)_{n \in \mathcal{A}}$ are independent whenever $\mathcal{A}$ and $-\mathcal{A}$ do not intersect ; $\left(A_{n}\right)_{n \in \mathbb{Z}^{d}}$ is independent of $\Phi ; \mathbb{E}\left[\sum_{\mathbb{Z}^{d}} A_{n}^{2}\right]<\infty ; A_{0}=0$ almost surely". Write

$$
S_{\mathcal{A}_{k}}(\omega, t)=\sum_{n \in \mathcal{A}_{k}} A_{n}(\omega) e^{i\left(\Phi_{n}(\omega)+n \cdot t\right)}
$$

for all $\omega$ in $\Omega$ and $t$ in $\mathbb{T}^{d}$. The following result can be easily deduced from Theorem 1.

Corollary 1. Under the hypotheses $\mathcal{H}_{1}^{* *}$ and $\mathcal{H}_{2}$, the the chain of equivalence of Theorem 1 holds with $A_{n}$ instead of $a_{n}$ and $S_{A_{k}}$ defined by (33).

Proof. To prove that, notice that $\mathbb{E}\left[\sum_{\mathbb{Z}^{d}} A_{n}^{2}\right]<\infty$ implies that $\left(A_{n}\right)_{n \in \mathbb{Z}^{d}}$ is almost surely square summable, and thus almost surely, Theorem 1 can be applied conditionally on $\mathcal{F}\left(A_{n}, n \in \mathbb{Z}^{d}\right)$, the $\sigma$-algebra generated by $\left(A_{n}\right)_{n \in \mathbb{Z}^{d}}$, since $\Phi$ is independent of $\mathcal{F}\left(A_{n}, n \in \mathbb{Z}^{d}\right)$.

Remark 5. This is of particular interest since Gaussian processes satisfy $\mathcal{H}_{1}^{* *}$ and $\mathcal{H}_{2}$.

Notice however that Hypothesis $\mathcal{H}_{2}$ cannot be much relaxed. As argued by Cohen and Cuny in [5], the symmetry assumption on $A_{n} e^{i \Phi_{n}}$ for each $n$ cannot be replaced by $\mathbb{E}\left[A_{n} e^{i \Phi_{n}}\right]=0$ for each $n$. 


\section{Properties of Random Phase Noise Processes}

Throughout this section, we assume both hypotheses $\mathcal{H}_{1}$ and $\mathcal{H}_{2}$ to hold. Moreover, we assume the equivalent hypotheses in Theorem 1 to hold, and thus the sample paths of the random phase noise field $X$ are almost surely continuous. Explicit conditions (e.g. on the coefficients $\left.\left(a_{n}\right)_{n \in \mathbb{Z}^{d}}\right)$ have been thoroughly studied in the case $d=1$, e.g. in Kahane [11], Chapter 7 .

\subsection{Stationarity.}

Proposition 4. A random phase noise (RPN) process $X$ is a centered second-order process, with covariance

$$
c_{X}(t)=\operatorname{Cov}(X(t+s), X(s))=\sum_{n \in \mathbb{Z}^{d}} a_{n}^{2} \cos (n \cdot t)
$$

for all $s$ and $t$ in $\mathbb{T}^{d}$ (weak stationarity). Moreover, $X$ is strongly stationary in the sense that $(X(t))_{t \in \mathbb{T}^{d}}$ and $(X(t+\tau))_{t \in \mathbb{T}^{d}}$ have the same law for any $\tau$ in $\mathbb{T}^{d}$.

Finally, the autocorrelation of $X$ defined as

$$
R_{X}(\tau)=\frac{1}{(2 \pi)^{d}} \int_{\mathbb{T}^{d}} X(t) X(t+\tau) d t, \tau \in \mathbb{T}^{d},
$$

is deterministic and a.s. equal to $c_{X}$.

Proof. For each $t$ in $\mathbb{T}^{d}, X(t)$ is the almost sure limit of a centered martingale $\left(X_{k}(t)\right)_{k \in \mathbb{N}}\left(X_{k}(t)=\right.$ $\sum_{n \in \mathcal{A}_{k}} a_{n} e^{i n \cdot t+\Phi_{n}}$ for any method of summation $\left.\left(\mathcal{A}_{k}\right)_{k \in \mathbb{N}}\right)$, that is bounded by $\sum_{n \in \mathbb{Z}^{d}} a_{n}^{2}$ in the space $L^{2}(\Omega)$, so it is a centered random variable in $L^{2}(\Omega)$. It follows that

$$
\mathbb{E}[X(s) X(t)]=\mathbb{E}[X(s) \overline{X(t)}]=\lim _{k \rightarrow+\infty} \mathbb{E}\left[X_{k}(s) \overline{X_{k}(t)}\right]=\sum_{n \in \mathbb{Z}^{d}} a_{n}^{2} e^{i n \cdot(s-t)}=\sum_{n \in \mathbb{Z}^{d}} a_{n}^{2} \cos (n \cdot(s-t))
$$

holds thanks to $\mathcal{H}_{1}$.

Recall that the Fourier coefficients of $t \mapsto X(t-\tau)$ are $\left(e^{i n \cdot \tau} \widehat{X}_{n}\right)_{n \in \mathbb{Z}^{d}}$. By the definition of $\widehat{X}_{n}$ and $\mathcal{H}_{2},\left(e^{i n \cdot \tau} \widehat{X}_{n}\right)_{n \in \mathbb{Z}^{d}}$ and $\left(\widehat{X}_{n}\right)_{n \in \mathbb{Z}^{d}}$ have the same finite dimensional law. Thus, $X$ and $X(\cdot-\tau)$ have the same finite dimensional law and the same law thanks to the almost sure continuity (Remark 1).

Finally, thanks to Parseval identity,

$$
\frac{1}{(2 \pi)^{d}} \int_{\mathbb{T}^{d}} X(t) X(t+\tau) d t=\frac{1}{(2 \pi)^{d}} \int_{\mathbb{T}^{d}} X(t) \overline{X(t+\tau)} d t=\sum_{n \in \mathbb{Z}^{d}} a_{n}^{2} \cos (n \cdot \tau)
$$

holds for all $\tau$ a.s., so we can conclude that $R_{X}=c_{X}$ a.s.

Hence, a single sample path contains enough information to fully determine the covariance and the law of the entire process, which can have various applications. For instance, one only needs one sample path to get as many independent sample paths with the same law. Interestingly, a secondorder process that has a deterministic autocorrelation also has deterministic Fourier modulus.

Proposition 5. Let $Y: \Omega \times \mathbb{T}^{d} \mapsto \mathbb{R}$ a centered process with sample paths almost surely in $\mathcal{C}^{0}\left(\mathbb{T}^{d}\right)$. Assume that there exists a (deterministic) continuous even function $\rho: \mathbb{T}^{d} \rightarrow \mathbb{R}$ satisfying

$$
R_{Y}=\rho
$$

almost everywhere, almost surely. Then, there exists a unique sequence or non-negative real numbers $\left(a_{n}\right)_{n \in \mathbb{Z}^{d}}$ and a random phase field $\Phi$ such that

$$
Y(\omega, t)=\sum_{n \in \mathbb{Z}^{d}} a_{n} e^{i\left(n \cdot t+\Phi_{n}(\omega)\right)}
$$

holds in $L^{2}\left(\mathbb{T}^{d}\right)$ almost surely. 
Proof. Almost surely, we can write

$$
Y(\omega, \cdot) \stackrel{L^{2}\left(\mathbb{T}^{d}\right)}{=} \sum_{n \in \mathbb{Z}^{d}} A_{n}(\omega) e^{i \Phi_{n}(\omega)} e_{n}
$$

for some random variables $\left(A_{n}\right)_{n \in \mathbb{Z}^{d}}$ and $\left(\Phi_{n}\right)_{n \in \mathbb{Z}^{d}}$, with $A_{n}$ chosen non-negative for all $n$. Thanks to Parseval identity, we can rewrite

$$
R_{Y(\omega)}(\tau)=\frac{1}{(2 \pi)^{d}} \int_{\mathbb{T}^{d}} Y(\omega, t) Y(\omega, t+\tau) d t=\sum_{n \in \mathbb{Z}^{d}} A_{n}(\omega)^{2} e^{i n \cdot \tau}
$$

and

$$
\rho(\tau)=\sum_{n \in \mathbb{Z}^{d}} b_{n} e^{i n \cdot \tau}
$$

for some non-negative Fourier coefficients $\left(b_{n}\right)_{n \in \mathbb{Z}^{d}}$ thanks to Herglotz Theorem. Take $a_{n}=\sqrt{b_{n}}$ for each $\mathrm{n}$, and conclude thanks to the uniqueness of Fourier coefficients.

Remark 6. The result also holds under the assumption that the sample paths are almost surely in $L^{2}\left(\mathbb{T}^{d}\right)$.

5.2. Marginal laws. The law of the marginal, say $X(0)=\sum_{n \in \mathbb{Z}^{d}} a_{n} \cos \left(\Phi_{n}\right)$, has already been studied by Blevins in [4] for series with a finite number of terms. We complete this study to fit our more general case of an infinite series that converges in $L^{2}(\Omega)$. Let us recall that in $\mathcal{H}_{1}$ we assume that $a_{0}=0$. Then, one can compute the normalized kurtosis. Indeed, $\mathbb{E}\left[|X(0)|^{2}\right]=\sum_{n \in \mathbb{Z}^{d}} a_{n}^{2}$ according to Proposition 4 and thus $\mathbb{E}\left[|X(0)|^{2}\right]^{2}=\left(\sum_{n \in \mathbb{Z}^{d}} a_{n}^{2}\right)^{2}$. Moreover recall that for $\Phi$ uniformly distributed in $\mathbb{R} / 2 \pi \mathbb{Z}, \mathbb{E}\left[\cos ^{2}(\Phi)\right]=\frac{1}{2}$ and $\mathbb{E}\left[\cos ^{4}(\Phi)\right]=\frac{3}{8}$, and thus for $\mathcal{A} \subset \mathbb{Z}^{d}$ such that $\mathcal{A} \cap-\mathcal{A}=\varnothing$ and $\mathcal{A} \cup-\mathcal{A}=\mathbb{Z}^{d} \backslash\{0\}\left(\right.$ so $\left.X(0)=2 \sum_{n \in \mathcal{A}} a_{n} \cos \left(\Phi_{n}\right)\right)$ ) and

$$
\begin{aligned}
\mathbb{E}\left[\left|\frac{X(0)}{2}\right|^{4}\right] & =\sum_{n \in \mathcal{A}} a_{n}^{4} \mathbb{E}\left[\cos \left(\Phi_{n}\right)^{4}\right]+3 \sum_{(l, m) \in \mathcal{A}^{2}, l \neq m} a_{l}^{2} a_{m}^{2} \mathbb{E}\left[\cos \left(\Phi_{l}\right)^{2}\right] \mathbb{E}\left[\cos \left(\Phi_{m}\right)^{2}\right] \\
& =\frac{3}{8} \sum_{n \in \mathcal{A}} a_{n}^{4}+\frac{3}{4} \sum_{(l, m) \in \mathcal{A}^{2}, l \neq m} a_{l}^{2} a_{m}^{2}=\frac{3}{4}\left(\sum_{n \in \mathcal{A}} a_{n}^{2}\right)^{2}-\frac{3}{8} \sum_{n \in \mathcal{A}} a_{n}^{4}
\end{aligned}
$$

so the kurtosis $\beta_{2}$ of $X(0)$ is given by

$$
\beta_{2}=\frac{\mathbb{E}\left[|X(0)|^{4}\right]}{\mathbb{E}\left[|X(0)|^{2}\right]^{2}}=3-\frac{3}{2} \frac{\sum_{n \in \mathcal{A}} a_{n}^{4}}{\left(\sum_{n \in \mathcal{A}} a_{n}^{2}\right)^{2}}=3-3 \frac{\sum_{n \in \mathbb{Z}^{d}} a_{n}^{4}}{\left(\sum_{n \in \mathbb{Z}^{d}} a_{n}^{2}\right)^{2}}<3,
$$

which proves that $X(0)$ is not Gaussian.

Remark 7. Actually $X(0)$ is not infinitely-divisible, and thus not Gaussian. Indeed, thanks to the independence hypothesis on $\Phi$ in $\mathcal{H}_{2}$, one easily checks that the characteristic function of $X(0)$ is the (maybe infinite) product

$$
\mathbb{E}\left[e^{i \xi X(0)}\right]=\prod_{n \in \mathcal{A}} \mathbb{E}\left[e^{i 2 a_{n} \xi \cos \left(\Phi_{n}\right)}\right]=\prod_{n \in \mathcal{A}} J_{0}\left(2 a_{n} \xi\right)
$$

with $J_{0}$ the Bessel function of the first kind, which admit zeroes on the real line. Hence, the characteristic function of $X(0)$ cannot be the characteristic function of an infinitely divisible random variable (see Theorem 5.3. p.108 of $[15]$ ).

Proposition 6. $X(0)$ is sub-Gaussian. More precisely, for all $\lambda$ in $\mathbb{R}$,

$$
\mathbb{E}\left[e^{\lambda X(0)}\right] \leq e^{\lambda^{2} \sum_{n \in \mathbb{Z}^{d}} a_{n}^{2}} .
$$


Proof. First, notice that a centered random variable $Y$ bounded by one is sub-Gaussian. Indeed, let $\lambda \in \mathbb{R}$, then $e^{\lambda Y} \leq \cosh (\lambda)+Y \sinh (\lambda)$ since $|Y| \leq 1$ and $x \mapsto e^{\lambda x}$ is convex. Then, using the fact that $Y$ is centered we get $\mathbb{E}\left(e^{\lambda Y}\right) \leq \cosh (\lambda) \leq e^{\lambda^{2} / 2}$. Now let $\left(\Phi_{n}\right)_{n \in \mathbb{Z}^{d}}$ be a a pure phase noise field. For a finite sum $X_{k}(0)=\sum_{|n|_{\infty} \leq k} a_{n} \cos \left(\Phi_{n}\right)$, thanks to the independence hypothesis for a subset $\mathcal{A} \subset \mathbb{Z}^{d}$ such that $\mathcal{A} \cap-\mathcal{A}=\varnothing$ and $\mathcal{A} \cup-\mathcal{A}=\mathbb{Z}^{d} \backslash\{0\}$

$$
\mathbb{E}\left[e^{\lambda X_{k}(0)}\right]=\prod_{n \in \mathcal{A},|n|_{\infty} \leq k} \mathbb{E}\left[e^{\lambda 2 a_{n} \cos \left(\Phi_{n}\right)}\right] \leq \prod_{n \in \mathcal{A},|n|_{\infty} \leq k} e^{\left(\lambda 2 a_{n}\right)^{2} / 2}=e^{\lambda^{2} \sum_{|n|_{\infty} \leq k} a_{n}^{2}}
$$

holds for all $\lambda \in \mathbb{R}$, since $\left(\cos \left(\Phi_{n}\right)\right)_{n \in \mathcal{A}}$ are independent centered random variables bounded by one.

For a general sum $X(0)=\sum_{n \in \mathbb{Z}^{d}} a_{n} \cos \left(\Phi_{n}\right)$ where $\left(a_{n}\right)_{n \in \mathbb{Z}^{d}}$ is a square summable family, let $\lambda$ be any real number and notice that $\mathbb{E}\left[e^{\lambda X_{k}(0)}\right] \leq e^{\lambda^{2} \sum_{|n|_{\infty} \leq k} a_{n}^{2}} \leq e^{\lambda^{2} \sum_{n \in \mathbb{Z}^{d}} a_{n}^{2}}$. Moreover $X_{k}(0) \rightarrow X(0)$ almost surely, thus $e^{\lambda X_{k}(0)} \rightarrow e^{\lambda X(0)}$ almost surely and we can apply Fatou's lemma and conclude that (46) holds.

Proposition 7. Assume that $\left(a_{n}\right)_{n \in \mathbb{Z}^{d}}$ is a family satisfying $\mathcal{H}_{1}$ and $\mathcal{H}_{2}$, such that

(1) there exists $n_{1}, n_{2}, n_{3}$ in $\mathbb{Z}^{d}$ with $a_{n_{1}} a_{n_{2}} a_{n_{3}} \neq 0$;

(2) $\left\{n_{1}, n_{2}, n_{3}\right\} \cap\left\{-n_{1},-n_{2},-n_{3}\right\}=\varnothing$.

Then $X(0)$ admits a density function that is uniformly continuous and bounded over $\mathbb{R}$.

The proof is postponed to Appendix. Interestingly, in the cases where only one or two coefficients are non-zero, the resulting Random Phase Noise process has an unbounded density function.

\section{ACKNOWLEDGMENTS}

We thank the anonymous referee for his useful comments, which allowed us to improve the quality of the manuscript.

\section{Appendix: Proofs}

6.1. Proof of Proposition 2. Proposition 2 is based on Proposition 13 pp. 55-56 in [11], we provide a proof for the sake of completeness. Let us first show a lemma, itself based on Proposition 12 p. 55 in Kahane [11].

Lemma 2. Let $\left(u_{k}\right)_{k \in \mathbb{N}}$ be a sequence in $\mathcal{C}^{0}\left(\mathbb{T}^{d}\right)$ with real or complex values, such that

$$
\underset{k}{\limsup }\left\|u_{k}\right\|_{\infty}>0 \text {. }
$$

Let $\left(\Psi_{k}\right)_{k \in \mathbb{N}}$ be a sequence of independent random variables uniformly distributed on $\mathbb{T}^{d}$. Then, almost surely, there exists $T$ (random) in $\mathbb{T}^{d}$ such that

$$
\limsup _{k}\left|u_{k}\left(T-\Psi_{k}\right)\right|>0 \text {. }
$$

Proof. Since $\lim \sup _{k}\left\|u_{k}\right\|_{\infty}>0$ by assumption, there exists both some $\eta>0$ and a subsequence $\left(k_{p}\right)_{p \in \mathbb{N}}$ such that

$$
\left\|u_{k_{p}}\right\|_{\infty}>\eta
$$

for all $p$. Thanks to continuity, $u_{k_{p}}(t)>\eta$ for $t$ in an open ball $B_{\infty}\left(t_{p}, \varepsilon_{p}\right)$. Thus, $\left|u_{k_{p}}\left(t-\Psi_{k_{p}}\right)\right|>\eta$ holds for $t$ in a random open ball $U_{p}:=B_{\infty}\left(T_{p}, \varepsilon_{p}\right)$ whose center is a random variable $T_{p}:=t_{p}+\Psi_{k_{p}}$ that is equidistributed on $\mathbb{T}^{d}$. Moreover $\left(T_{p}\right)_{p \in \mathbb{N}}$ is i.i.d. since $\left(\Psi_{k}\right)_{k \in \mathbb{N}}$ is assumed to be i.i.d.

Now, let us show that $\lim \sup _{p} U_{p}$ is almost surely non-empty (it can be shown that it is actually almost surely dense). Let $t$ be any point in $\mathbb{T}^{d}, \varepsilon>0$ be a positive number and denote $U:=B_{\infty}(t, \varepsilon)$. For each $p, \mathbb{P}\left(U_{p} \cap U \neq \varnothing\right) \geq \mathbb{P}\left(T_{p} \in U\right)=\operatorname{vol}(U) /(2 \pi)^{d}$ since $T_{p}$ is equidistributed on $\mathbb{T}^{d}$. Thus $\sum_{p} \mathbb{P}\left(U_{p} \cap U \neq \varnothing\right)=\infty$, and since the events $\left\{\omega \mid U_{p}(\omega) \cap U \neq \varnothing\right\}$ are independent, it follows thanks to Borel-Cantelli Lemma that almost surely $U_{p} \cap U \neq \varnothing$ happens for infinitely many $p$. Thus, almost surely, $\lim \sup _{p} U_{p} \neq \varnothing$.

Let us pick some random $T$ in $\lim \sup _{p} U_{p}$ and notice that $\lim \sup _{p}\left|u_{k_{p}}\left(T-\Psi_{k_{p}}\right)\right|>\eta$ almost surely since $T$ belongs to infinitely many $U_{p}$. This concludes the proof. 
Let us now prove Proposition 2.

First, let us recall that since for all $k$ the random variable $Y_{k}\left(\right.$ in $\left.\mathcal{C}^{0}\left(\mathbb{T}^{d}\right)\right)$ is assumed to be symmetric (by 1.), Itô-Nisio Theorem applies. Hence, the series $\sum_{k} Y_{k}$ converges almost surely in $\mathcal{C}^{0}\left(\mathbb{T}^{d}\right)$ if and only if any subsequence converges in $\mathcal{C}^{0}\left(\mathbb{T}^{d}\right)$ in probability.

Let us assume that the conclusion does not hold. Then, there must exist some $\eta>0$ and two sequences of integers $\left(k_{p}\right)_{p \in \mathbb{N}}$ and $\left(k_{p}^{\prime}\right)_{p \in \mathbb{N}}$ such that $k_{p}<k_{p}^{\prime}<k_{p+1}$ for each $p$ and

$$
\mathbb{P}\left(\left\|\sum_{k=k_{p}+1}^{k_{p+1}} Y_{k}\right\|_{\infty}>\eta\right)>\eta
$$

for all $p$.

Let $\left(\Omega^{\prime}, \mathbb{P}_{\Omega^{\prime}}\right)$ denote the probability space $\Omega \times \Omega_{\Psi}$ with $\mathbb{P}_{\Omega^{\prime}}=\mathbb{P}_{\Omega} \otimes \mathbb{P}_{\Psi}$, where $\Omega_{\Psi}$ is a probability space in which there is a sequence $\left(\Psi_{n}\right)_{n \in \mathbb{N}}$ of i.i.d. random variables equidistributed on $\mathbb{T}^{d}$. Let us write $Z_{p}=\sum_{k=k_{p}+1}^{k_{p+1}} Y_{k}$ for all $p$, and let us consider the series of functions $\sum Z_{p}(\cdot)$ and $\sum Z_{p}\left(\cdot-\Psi_{p}\right)$ as random series (in the probability space $\Omega^{\prime}$ ) of elements in $\mathcal{C}^{0}\left(\mathbb{T}^{d}\right)$. Since the $Y_{k}(k$ in $\mathbb{N}$ ) are independent and symmetrical (by 1.), so are the $Z_{p}(p$ in $\mathbb{N})$. Since for all $k, Y_{k}$ and its translates have the same law (by 2.), $Z_{p}$ and $Z_{p}\left(\cdot-\Psi_{p}\right)$ have the same law for each $p$. Moreover, since the sequence $\left(\sum_{k \leq l} Y_{k}\right)_{l \in \mathbb{N}}$ is almost surely bounded in $\mathcal{C}^{0}\left(\mathbb{T}^{d}\right)$ (by 3.), the series of functions $\sum_{p} Z_{p}$ is also almost surely bounded.

Moreover $\left(Z_{p}\right)_{p \in \mathbb{N}}$ is a sequence of independent variables and $\mathbb{P}_{\Omega}\left(\left\|Z_{p}\right\|_{\infty}>\eta\right)>\eta$ for each $p$, and thus $\sum_{p} \mathbb{P}_{\Omega}\left(\left\|Z_{p}\right\|_{\infty}>\eta\right)=\infty$. Hence Borel-Cantelli lemma applies and, almost surely (in $\Omega$ ), $\lim \sup _{p}\left\|Z_{p}\right\|_{\infty}>\eta$. As a consequence, almost surely (in $\Omega^{\prime}$ ), $\lim \sup _{p}\left\|Z_{p}\right\|_{\infty}>\eta$. Lemma 2 yields that almost surely in $\Omega^{\prime}, \lim \sup _{p}\left|Z_{p}\left(T-\Psi_{p}\right)\right|>0$ for some (random) $T$ in $\mathbb{T}^{d}$.

Let us introduce another probability space $\Omega^{\prime \prime}=\Omega^{\prime} \times \Omega_{\varepsilon}\left(\mathbb{P}_{\Omega^{\prime \prime}}=\mathbb{P}_{\Omega} \otimes \mathbb{P}_{\Psi} \otimes \mathbb{P}_{\varepsilon}\right)$ and a Rademacher sequence $\left(\varepsilon_{p}\right)_{k \in \mathbb{N}}$. We now consider the random series of functions $\sum_{p} Z_{p}\left(t-\Psi_{p}\right)$ and $\sum_{p} \varepsilon_{p} Z_{p}\left(t-\Psi_{p}\right)$ on the space $\Omega^{\prime \prime}$. Since the random functions $Z_{p}$ are symmetric, the partial sums have the same law in $\Omega^{\prime \prime}$. Moreover, since $\lim \sup _{p}\left|Z_{p}\left(T-\Psi_{p}\right)\right|>0$ almost surely (in $\Omega^{\prime \prime}$ ),

$$
\sum_{p=1}^{\infty}\left|Z_{p}\left(T-\Psi_{p}\right)\right|^{2}=\infty
$$

holds almost surely (in $\Omega^{\prime \prime}$ ). Thus the sequence $\left(\sum_{1}^{N} \varepsilon_{p} Z_{p}\left(t-\Psi_{p}\right)\right)_{N \in \mathbb{N}}$ is almost surely (in $\left.\Omega^{\prime \prime}\right)$ not bounded for some (random) $T$, thanks to a classic consequence of Paley-Zygmund inequalities (see [11] Theorem 1 p. 54).

To conclude, recall that $\left(\sum_{p=1}^{N} Z_{p}\right)_{N \in \mathbb{N}}$ is assumed to be almost surely bounded in $\mathcal{C}^{0}\left(\mathbb{T}^{d}\right)$ (in the probability space $\Omega$ and thus also in $\left.\Omega^{\prime \prime}\right)$. Finally, notice that $Z_{p}$ and $\varepsilon_{p} Z_{p}\left(\cdot-\Psi_{p}\right)$ have the same law in $\Omega^{\prime \prime}$ and thus $\left(\varepsilon_{p} \sum_{p=1}^{N} Z_{p}\left(\cdot-\Psi_{p}\right)\right)_{N \in \mathbb{N}}$ must also be almost surely bounded, which is a contradiction.

6.2. Proof of Proposition 3. The proof is a generalization to $d \geq 2$ of Kahane's [11] Proposition 5 p. 49. We begin with a lemma that gives a Bernstein's inequality for a multivariate trigonometric polynomial. In the following result, $\|\cdot\|$ denotes the norm on linear forms induced by $|\cdot|_{\infty}$ the maximum norm over $\mathbb{R}^{d}$, and $\nabla P(t)$ denotes the gradient of the trigonometric polynomial $P$ at point $t$.

Lemma 3. Let $K$ be some positive integer and $P$ a trigonometric polynomial on $\mathbb{T}^{d}$ with max-degree less than $K$ defined by $P(t)=\sum_{|n|_{\infty} \leq K} b_{n} e^{i\left(n \cdot t+\phi_{n}\right)}$ for all $t$ in $\mathbb{T}^{d}$. Then

$$
\sup _{t \in \mathbb{T}^{d}}\|\nabla P(t)\| \leq K\|P\|_{\infty} \text {. }
$$

Proof. Let us denote $\left(\theta_{k}\right)_{1 \leq k \leq d}$ the canonical basis of $\mathbb{R}^{d}$. Let us introduce for $t \in \mathbb{T}^{d}, 1 \leq k \leq d$, the real trigonometric polynomial $Q_{k}(u)=P\left(t+u \theta_{k}\right)$. According to Bernstein's inequality one has

$$
\left\|Q_{k}^{\prime}\right\|_{\infty} \leq \max _{|n|_{\infty} \leq K}\left|n \cdot \theta_{k}\right|\left\|Q_{k}\right\|_{\infty},
$$


which involves that

and proves (53).

$$
\left|\frac{\partial P}{\partial t_{k}}(t)\right|=\left|Q_{k}^{\prime}(0)\right| \leq K\|P\|_{\infty}
$$

For completeness, let us mention the following variant of Lemma 3 suggested by a reviewer. It establishes a similar result for the $\ell^{2}$ norm of $\nabla P$ when the usual (not max) degree of $P$ is used (that is, $|n|_{1}$ when $\left.P(t)=\sum_{n} a_{n} e^{i n \cdot t}\right)$.

Let $K$ be some positive integer and $P$ a trigonometric polynomial on $\mathbb{T}^{d}$ with degree less than $K$, defined by $P(t)=\sum_{|n|_{1} \leq K} b_{n} e^{i\left(n \cdot t+\phi_{n}\right)}$ for all $t$ in $\mathbb{T}^{d}$. Then

$$
\|\nabla P(t)\|_{\ell^{2}} \leq K\|P\|_{\infty} .
$$

Proof: Let $\omega=\left(\varepsilon_{1}, \ldots, \varepsilon_{d}\right) \in\{-1,1\}^{d}$, then for fixed $t$,

$$
Q_{\omega}(u)=P(t+u \omega)
$$

is a trigonometric poynomial of degree less than $K$ in one variable (precisely because $|n|_{1} \leq K$ for $n$ in the spectrum of $P$ ), with sup-norm dominated by that of $P$. The usual Bernstein inequaliy in one variable then gives

$$
\left|Q_{\omega}^{\prime}(0)\right|=\left|\sum_{k=1}^{d} \varepsilon_{k} \frac{\partial P}{\partial t_{k}}(t)\right| \leq K\|P\|_{\infty} .
$$

Squaring and taking the average over all choices of signs $\varepsilon_{j}$ (using Rademacher variables if one wishes), we obtain the desired inequality (54).

We now turn to the proof of Proposition 3. Let $\varepsilon \geq 2 \pi / q$ and $t \in \mathbb{T}^{d}$. The function $s \mapsto|P(s)|$ is $2 \pi / q$-periodic on each component. Indeed, write $\mathcal{E}=\{l+q j\}_{j \in \mathcal{E}^{\prime}}\left(\mathcal{E}^{\prime} \subset \mathbb{Z}^{d}\right)$ and notice that

$$
|P(s)|=\left|\sum_{j \in \mathcal{E}^{\prime}} b_{l+q j} e^{i\left((l+q j) \cdot s+\phi_{n}\right)}\right|=\left|e^{i(l \cdot s)} \sum_{j \in \mathcal{E}^{\prime}} b_{l+q j} e^{i\left(q j \cdot s+\phi_{n}\right)}\right|=\left|\sum_{j \in \mathcal{E}^{\prime}} b_{l+q j} e^{i\left(q j \cdot s+\phi_{n}\right)}\right|
$$

for each $s$ in $\mathbb{T}^{d}$. Let $t^{\prime}$ in $\mathbb{T}^{d}$ be such that $|P|$ achieves its global maximum $\|P\|_{\infty}$ at point $t^{\prime}$ that may be assumed to be in $\mathbb{B}_{\infty}(t, \varepsilon / 2)$, thanks to the $2 \pi / q$-periodicity of $s \mapsto|P(s)|$. For all $s$ in $\mathbb{T}^{d}$,

$$
\left|P(s)-P\left(t^{\prime}\right)\right|=P\left(t^{\prime}\right)-P(s)=\|P\|_{\infty}-P(s) \leq \sup _{u}\|\nabla P(u)\|\left|t^{\prime}-s\right| \leq K\|P\|_{\infty}\left|t^{\prime}-s\right|
$$

thanks to Lemma 3 , and thus

$$
P(s) \geq\|P\|_{\infty} / 2
$$

for all $s$ in $\mathbb{B}_{\infty}\left(t^{\prime}, 1 /(2 K)\right)=\mathbb{B}_{\infty}\left(t^{\prime}, \varepsilon^{\prime}\right)$. Since $K \geq \frac{q}{2 \pi}$, $\varepsilon^{\prime}$ satisfies

$$
\varepsilon^{\prime} \leq \pi / q \leq \varepsilon / 2
$$

and thus $s \mapsto|P(s)|$ achieves its global maximum $\|P\|_{\infty}$ on a point $t^{\prime}$ such that $\mathbb{B}_{\infty}\left(t^{\prime}, \varepsilon^{\prime}\right) \subset \mathbb{B}_{\infty}(t, \varepsilon)$.

6.3. Proof of Lemma 4. Define $\mathcal{B}_{+}=\mathcal{B} \cap\left(\mathbb{N} \times \mathbb{Z}^{d-1}\right)$ and $\mathcal{B}_{-}=\mathcal{B} \cap\left(-\mathbb{N} \times \mathbb{Z}^{d-1}\right)$. Notice that, with non-zero probability, at least one of the sequences among $\left(\sum_{n \in \mathcal{B}_{+} \cap \mathcal{A}_{k}} a_{n} e^{i\left(n \cdot T+\Phi_{n}\right)}\right)_{k \in \mathbb{N}}$ and $\left(\sum_{n \in \mathcal{B}_{-} \cap \mathcal{A}_{k}} a_{n} e^{i\left(n \cdot T+\Phi_{n}\right)}\right)_{k \in \mathbb{N}}$ diverges. Thus, we can define $\mathcal{B}^{\prime}$, a deterministic subset of $\mathbb{Z}^{d}$ either equal to $\mathcal{B}_{+}$or equal to $-\left(\mathcal{B}_{-}\right)$, such that, with non-zero probability, the sequence $\left(Z_{k}\right)_{k \in \mathbb{N}}$ defined by

$$
Z_{k}=\sum_{n \in \mathcal{A}_{k} \cap \mathcal{B}^{\prime}} a_{n} e^{i\left(n \cdot T+\Phi_{n}\right)}
$$

diverges. Define $X_{k}:=\operatorname{Re}\left(Z_{k}\right)=\sum_{n \in \mathcal{A}_{k} \cap \mathcal{B}^{\prime}} a_{n} \cos \left(n \cdot T+\Phi_{n}\right)$ and $Y_{k}:=\operatorname{Im}\left(Z_{k}\right)=\sum_{n \in \mathcal{A}_{k} \cap \mathcal{B}^{\prime}} a_{n} \sin (n$. $\left.T+\Phi_{n}\right)$ for all $k$. With non-zero probability $\left(X_{k}\right)_{k \in \mathbb{N}}$ or $\left(Y_{k}\right)_{k \in \mathbb{N}}$ diverges. Let us define the events

$$
E_{\text {div }}^{\text {cos }}:=\left\{\omega \mid \text { the sequence }\left(X_{k}\right)_{k \in \mathbb{N}} \text { diverges }\right\}
$$

and

$$
E_{\mathrm{div}}^{\sin }:=\left\{\omega \mid \text { the sequence }\left(Y_{k}\right)_{k \in \mathbb{N}} \text { diverges }\right\}
$$


The event

$$
E_{\text {div }}:=E_{\text {div }}^{\cos } \cup E_{\text {div }}^{\text {sin }}
$$

happens with non-zero probability.

Since $\left(\Phi_{n}\right)_{n \in \mathbb{N} \times \mathbb{Z}^{d-1}}$ and $\left(\Phi_{n}+\frac{\pi}{2}\right)_{n \in \mathbb{N} \times \mathbb{Z}^{d-1}}$ have the same law (direct consequence of $\mathcal{H}_{2}$ ), the events $E_{\text {div }}^{\text {cos }}$ and $E_{\text {div }}^{\text {sin }}$ have the same probability. Thus, the probability of the event $E_{\text {div }}^{\text {cos }}$ is non-zero. We conclude by defining $\mathcal{B}^{*}=\mathcal{B}^{\prime} \cup\left(-\mathcal{B}^{\prime}\right)$ and noticing that $\sum_{n \in \mathcal{A}_{k} \cap \mathcal{B}^{*}} a_{n} \cos \left(n \cdot T+\Phi_{n}\right)=2 X_{k}$ for each $k$.

6.4. Proof of Proposition 7. Since the law $\mathbb{P}_{X(0)}$ of the limit $X(0)$ does not depend on a method of summation (Proposition 1), let us pick one ordering in $\mathbb{N} \times \mathbb{Z}^{d-1},\left(n_{k}\right)_{k \in \mathbb{N}}$, and rewrite $b_{k}:=a_{n_{k}}$ for each $k \in \mathbb{N}$. For simplicity, let us assume that $b_{k} \neq 0$ for each $k$. We may write

$$
X(0)=\sum_{k=1}^{3} b_{k} \cos \left(\Phi_{n_{k}}\right)+\sum_{k=4}^{+\infty} b_{k} \cos \left(\Phi_{n_{k}}\right)=Y+Z .
$$

By independence one has $\mathbb{P}_{X(0)}=\mathbb{P}_{Y} * \mathbb{P}_{Z}$. Since the convolution of a probability measure with an absolutely continuous measure with uniformly continuous bounded density remains an absolutely continuous measure with uniformly continuous bounded density, it is sufficient to prove that $Y=$ $\sum_{k=1}^{3} b_{k} \cos \left(\Phi_{n_{k}}\right)$ admits a uniformly continuous bounded density.

Let us recall that the density function of $b \cos (\Phi)$, where $\Phi$ is a uniform random variable over $\mathbb{R} / 2 \pi \mathbb{Z}$ and $b$ a non-zero real number, is $t \mapsto \frac{1}{b} f\left(\frac{t}{b}\right)$, with $f(t)=\mathbb{1}_{(-1,1)} \frac{1}{\pi \sqrt{1-t^{2}}}$. Moreover, one easily checks that $f$ is in $L^{p}(\mathbb{R})$ for every $p$ in $[1,2)$ and hence so is the density function of the random variable $b \cos (\Phi)$. Let $f_{1}$ (resp. $f_{2}, f_{3}$ ) denote the density function of $b_{1} \cos \left(\Phi_{n_{1}}\right)$ (resp. $b_{2} \cos \left(\Phi_{n_{2}}\right)$, $b_{3} \cos \left(\Phi_{n_{3}}\right)$ ) such that the density of $Y$ is given by $f_{1} * f_{2} * f_{3}$. Recall that Young's inequalities (see e.g. [14] p. 99) state that if $p, q, r$ are in $[1, \infty]$, such that

$$
\frac{1}{p}+\frac{1}{q}=1+\frac{1}{r}
$$

and if $f \in L^{p}(\mathbb{R})$ and $g \in L^{q}(\mathbb{R})$ one has $f * g \in L^{r}(\mathbb{R})$ with $\|f * g\|_{r} \leq\|f\|_{p}\|g\|_{q}$. It follows that the convolution $f_{1} * f_{2}$ belongs to $L^{p}(\mathbb{R})$ for all $p$ in $[1, \infty)$, since $f_{1}$ and $f_{2}$ are in $L^{p}(\mathbb{R})$ for every $p$ in $[1,2)$. In particular it belongs to $L^{3}(\mathbb{R})$. Moreover, $f_{3}$ belongs to $L^{3 / 2}(\mathbb{R})$ and since 3 and $3 / 2$ are conjugate exponents $(1 / 3+2 / 3=1),\left(f_{1} * f_{2}\right) * f_{3}$ is uniformly continuous and bounded (see $e . g$. [14] p. 70).

\section{REFERENCES}

[1] J. M. Ash and G. V. Welland. Convergence, uniqueness, and summability of multiple trigonometric series. Trans. Amer. Math. Soc., 163:401-436, 1972.

[2] H. Berens and Y. Xu. Fejèr Means for Multivariate Fourier Series. Mathematische Zeitschrift, 221:449-465, 1996.

[3] P. Billard. Séries de Fourier Aléatoirement Bornées, Continues, Uniformément Convergentes. Studia Mathematica, 22:310-330, 1963.

[4] R. D. Blevins. Probability density of finite Fourier series with random phases. J. Sound Vibration, 208(4):617-652, 1997.

[5] G. Cohen and C. Cuny. On Billard's Theorem for Random Fourier Series. Bull. Pol. Acad. Sci. Math., 53:39-53, 2005.

[6] C. Fefferman. On the Convergence of Multiple Fourier Series. Bulletin of the American Mathematical Society, $77(5): 744-745,1971$.

[7] C. Fefferman. On the Divergence of Multiple Fourier Series. Bulletin of the American Mathematical Society, $77(2): 191-195,1971$.

[8] A. Figà-Talamanca. Bounded and Continuous Random Fourier Series on Noncommutative Groups. Proceedings of the AMS, 22:573-578, 1969.

[9] B. Galerne, Y. Gousseau, and J.-M. Morel. Random Phase Textures: Theory and Synthesis. IEEE Transactions on Image Processing, 20(1):257-267, 2011.

[10] K. Itó and M. Nisio. On the Convergence of Sums of Independent Banach Space Valued Random Variables. Osaka J. Math, 5:35-48, 1968. 
[11] J.-P. Kahane. Some Random Series of Functions. Cambridge studies in advanced mathematics. Cambridge University Press, 1994.

[12] Y. Katznelson. Sur les Ensembles de Divergence des Séries Trigonométriques. Studia Math., 26:301-304, 1966.

[13] M. Ledoux and M. Talagrand. Probability in Banach Spaces. Ergebnisse der Mathematik und ihrer Grenzgebiete. Springer, 1991.

[14] E. H. Lieb and M. Loss. Analysis: Second Edition. Graduate Studies in Mathematics. AMS, 2001.

[15] E. Lukacs. Characteristic functions. Hafner Publishing Co., New York, 1970. Second edition, revised and enlarged.

[16] M. B. Marcus and G. Pisier. Random Fourier Series with Applications to Harmonic Analysis. Anals of Mathematics Studies. Princeton University Press, 1981.

[17] R. Paley and A. Zygmund. On Some Series of Functions, part I. Proc. Cambridge Philo. Soc., 26:337-357, 1930.

[18] R. Paley and A. Zygmund. On Some Series of Functions, part II. Proc. Cambridge Philo. Soc., 26:458-474, 1930.

[19] R. Paley and A. Zygmund. On Some Series of Functions, part III. Proc. Cambridge Philo. Soc., 28:190-205, 1932.

[20] N. Tevzadze. On the Convergence of Double Fourier Series of Quadratic Summable Functions. Soobsc. Akad. Nauk Gruzin. SSR, 77(5):277-279, 1970.

[21] J. van Wijk. Spot Noise Texture Synthesis for Data Visualization. SIGGRAPH Comput. Graph., 25(4):309-318, 1991.

${ }^{1}$ Map5, Umr CNRS 8145, Université Paris Descartes, Sorbonne Paris Cité, 45 rue des Saints-Pères, 75006 PARIS, France.

${ }^{2}$ LMA, UMr CNRs 7348, Université de Poitiers, Boulevard Marie et Pierre Curie, 86962 Chasseneuil, FRANCE.

E-mail address: [samuel.ronsin,lionel.moisan] @parisdescartes.fr, hermine.bierme@math.univ-poitiers.fr 